\section{Movimientos sociales, agroecología y soberanía alimentaria. Un acercamiento al proyecto pedagógico de la Universidad Campesina- Sistemas Universitarios Rurales Indoamericanos, Santiago del Estero, Argentina}

\author{
Mabel García \\ Universidad de Buenos Aires, Argentina. \\ Instituto de Ecología y Desarrollo Sustentable, \\ Consejo Nacional de Investigaciones \\ Científicas y Técnicas-Universidad Nacional \\ de Luján, Argentina. Asociación Argentino \\ Uruguaya de Economía Ecológica. \\ mabelg@agro.uba.ar \\ (iD) orcid.org/0000-0003-4280-2557
}

RECEPCIÓN: 29/04/21

ACEPTACIÓN FINAL: 07/06/21

\author{
Andrea Estefanía Figueroa \\ aefigueroa@agro.uba.ar \\ (iD) orcid.org/0000-0003-1329-5816 \\ Samanta Villa \\ villa@agro.uba.ar \\ (iD) orcid.org/0000-0002-4490-180X
}

Ambiente y extensión universitaria / Perspectivas

\section{다(1)(2)}

Noelia Calefato

calefato@agro.uba.ar

(iD) orcid.org/0000-0001-6536-5826

Universidad de Buenos Aires,

Argentina.

\author{
Social Movements, Agroecology and \\ Food Sovereignty. An approach to the \\ pedagogical project of the Peasant \\ University-Indo-American Rural University \\ Systems, Santiago del Estero, Argentina
}

\section{Abstract}

En América Latina, durante las últimas décadas los movimientos sociales han trabajado para satisfacer necesidades alimentarias en un contexto donde las transformaciones rurales se han basado en estrategias que gobiernan desde lo global los sistemas productivos locales con el objetivo de acumular capital. La Universidad Campesina-Sistemas Universitarios Rurales Indoamericanos es un proyecto pedagógico de educación popular del Movimiento Campesino de Santiago del Estero-Vía Campesina, enmarcado a su vez en el proyecto de los Institutos Agroecológicos Latinoamericanos de Escuelas de Formación en Agroecológica de la VC para la construcción de un modelo contrahegemónico. El objetivo de este artículo es reflexionar sobre los aportes de dicha Universidad Campesina al movimiento indígena-campesino y a la construcción de la soberanía alimentaria. El abordaje metodológico es la InvestigaciónAcción Participativa. La contribución de la Universidad Campesina trasciende su zona de influencia y su propuesta pedagógica enfrenta desafíos que podrían superarse articulando con distintas instituciones.

Palabras clave: agroecología; soberanía alimentaria; movimientos sociales; extensión crítica; Universidad Campesina-Sistemas Universitarios Rurales Indoamericanos; UNICAM SURI.
In Latin America, during the last decades, social movements have worked to satisfy local food needs in a context where rural transformations have been based on strategies that govern local productive systems from the global with the objective of accumulating capital. The Peasant University-Indo-American Rural University Systems is a pedagogical project of popular education of the Peasant Movement of Santiago del Estero-Vía Campesina, framed in the project of the Latin American Agroecological Institutes of Agroecological Training Schools of the VC, for the construction of a counter-hegemonic model. The objective of this article is to reflect on the contributions of the Peasant University to the indigenous-peasant movement and how it contributes to the construction of food sovereignty. The methodological approach is Participatory Action Research. The contributions of The Peasant University transcend its area of influence and its pedagogical proposal faces challenges that could be faced by articulating with different institutions.

Keywords: agroecology; food sovereignty; social movements; emancipatory critical extensionism; Peasant University-IndoAmerican Rural University Systems; UNICAM SURI.

\section{Movimentos Sociais, Agroecologia e Soberania Alimentar. Uma aproximação ao projeto pedagógico da Universidade Campesina-Sistemas Universitarios Indoamericanos Rurales, Santiago del Estero, Argentina}

\section{Resumo}

Na América Latina, nas últimas décadas, os movimentos sociais trabalharam para atender às necessidades alimentares locais em um contexto em que as transformações do campo se basearam em estratégias que regem os sistemas produtivos locais a partir do global com o objetivo de acumular capital. A Universidade Campesina-Sistemas Universitarios Rurales Indoamericanos —UNICAM SURI- é um projeto pedagógico de educação popular do Movimento Camponês de Santiago del Estero-Via Campesina, enquadrado no projeto dos Institutos Agroecológicos Latino-Americanos de Escolas de Formação Agroecológica do VC, para a construção de um modelo contrahegemônico. O objetivo deste artigo é refletir sobre as contribuições da UNICAM-SURI para o movimento indígena-camponês e como contribui para a construção da soberania alimentar. A abordagem metodológica é a Pesquisa-Ação Participativa. As contribuições da UNICAM SURI transcendem sua área de influência e sua proposta pedagógica enfrenta desafios que poderiam ser enfrentados em articulação com diferentes instituições.

Palavras-chave: agroecologia; soberania alimentar; movimentos sociais; extensão crítica; Universidade Campesina-Sistemas Universitarios Indoamericanos Rurales; UNICAM SURI.

Para citación de este artículo: García, M.; Figueroa, E. A., Villa, S. y Calefato, N. (2021). Movimientos sociales, agroecología y soberanía alimentaria. Un acercamiento al proyecto pedagógico de la Universidad Campesina-Sistemas Universitarios Rurales Indoamericanos, Santiago del Estero, Argentina. +E: Revista de Extensión Universitaria, 11(14), e0006. doi: 10.14409/extension.2021.14.Ene-Jun.e0006 


\section{Introducción ${ }^{1}$}

El sistema agroalimentario mundial, dentro del actual contexto económico capitalista, ha ido configurando la agricultura y la alimentación, sometiéndolas a un marcado proceso de industrialización y globalización (FAO, 2017). Desde el último cuarto del siglo XX se ha acelerado en América Latina un conjunto de transformaciones agrarias que, mientras se consolida la llamada Revolución Verde y el discurso de la seguridad alimentaria, han puesto de manifiesto procesos de concentración y centralización del capital agroindustrial. Podemos mencionar procesos como el de concentración de la producción agropecuaria, de consolidación de formas de articulación de los productores agrarios con los complejos agroindustriales donde las grandes corporaciones transnacionales toman las principales decisiones, la aceleración de la mercantilización de los productos primarios y la expulsión de pequeños y medianos productores (Teubal, 2002). Sin embargo, el llamado "imperio corporativo alimentario" (McMichael, 2005; Ploeg, 2010), a la hora de acumular capital y crecer, no respeta límites ecológicos ni sociales. Mediante la conformación de plataformas o enclaves agroexportadores, basados en la extracción intensiva de recursos y apropiación de riqueza de los sistemas locales, gobiernan la dinámica interior de esos sistemas productivos (Etxezarreta, 2006). Con otro enfoque, existen numerosos trabajos que estudiaron el efecto negativo de estas transformaciones sobre la salud y bienestar humano, como las pulverizaciones, la presencia de plaguicidas en los alimentos, la reducción de la biodiversidad, la contaminación de agua y suelo, etc. (Di Ciocco et al., 2017). Por otro lado, Svampa (2012) estudia los conflictos ambientales que, en situaciones de asimetría de poder, se manifiestan en el enfrentamiento de actores, intereses y valores divergentes. Estos conflictos ponen sobre el tapete distintas concepciones y modelos de desarrollo (García et al., 2018).

En Argentina, según el enfoque de Salizzi (2013), la dinámica expansiva del capital agrario evolucionó a través de la "agriculturización" del área pampeana (1970-1990) y el boom de la soja transgénica (1990-2001). La agriculturización pampeana se manifiesta en el uso creciente y continuo del suelo para la agricultura donde se sustituyen usos ganaderos o mixtos (agrícolo-ganadero). Principalmente a raíz de las innovaciones tecnológicas —combinación de agroquímicos, fertilizantes y semillas híbridas- surgen procesos de intensificación de las tradicionales producciones pampeanas en términos de capital/hectárea. También se intensifica el uso de la tierra al elevar su productividad por hectárea. Este proceso ha dado como resultado una agricultura de capital intensiva asociada a innovaciones tecnológicas con alta concentración de la provisión de insumos y de capital (García, 2007). En este contexto, la diversidad de cultivos y la cantidad de producción destinados al abastecimiento de alimentos para la población se redujeron frente al avance de la superficie sembrada con soja para exportación, lo cual genera, de manera indirecta, el aumento de la canasta básica (Zeolla, 2012). A su vez, el modelo de producción agropecuaria orientado al mercado externo es insumodependiente, se basa en la producción a gran escala y en el desarrollo de un monocultivo transgénico como es la soja (Domínguez y Sabatino, 2006). Por ello, a los productores pampeanos e inversionistas de otros sectores de la economía, con el objetivo de reinvertir las ganancias obtenidas por la intensificación productiva y otras formas de concentración

1) En 2019 , versiones parciales y preliminares de este artículo fueron presentadas como ponencias en las XI Jornadas Interdisciplinarias de Estudios Agrarios y Agroindustriales, Facultad de Ciencias Económicas, Universidad de Buenos Aires. 
económica, se les plantearon dos posibilidades: intensificar aún más su producción en la región Pampeana o comprar tierras baratas en la región del Gran Chaco Argentino (GCA), ${ }^{2}$ y habilitarlas para la agricultura mediante talas rasas o "desmontes" de sistemas boscosos (Navarrete et al., 2005). Esta segunda opción captó el interés de numerosos empresarios y empresas agropecuarias provenientes sobre todo del área pampeana pero también de otras provincias (De Dios 2006; García, 2015). Las regiones periféricas adoptaron prácticas y tecnologías propias del modelo agropecuario pampeano sin considerar las diferencias edafológicas y climáticas locales (Morello, Pengue y Rodríguez, 2005; Navarrete et al., 2005). Además, es imprescindible tener en cuenta que las áreas extrapampeanas se inscriben en una problemática diferente ya que la situación irregular de tenencia de la tierra que presentan muchos pequeños productores, junto con la dificultad para el apoyo a la titulación de tierras y la ausencia de infraestructura básica, es un problema que se agravó cuando la producción agrícola valorizó la tierra ocupada por estos productores (García y Puppi, 2007; Paz y Jara, 2013; García et al., 2016). En consecuencia, la expansión motorizada por empresas capitalizadas resultó en muchos casos en la expulsión de los ocupantes históricos (De Dios, 2012). Algunos sectores de la población, los pequeños campesinos y parte de la población indígena, que residían sobre la base de los recursos que proveía el monte, tuvieron que trasladarse. Sin embargo, en la región del GCA la agricultura familiar ha logrado persistir atravesada por dificultades de distinta índole, tanto comerciales, como ambientales y jurídicas sobre la tenencia de la tierra (Román, 2014). El campesinado, como forma de la agricultura familiar, tiene una fuerte participación en la estructura agraria del GCA; dado que es una de las regiones con mayor población campesina e indígena del país, presenta una gran heterogeneidad socioproductiva y cultural que se traslada a la configuración del espacio rural, donde se ponen de manifiesto los conflictos por las formas de acceso y control de los diferentes recursos (García et al., 2018; Svampa, 2012). Estas disputas dan lugar para el análisis de vías y posibilidades de construcción contrahegemónica, como la producción agroecológica, paradigma emergente que intenta posicionarse como una alternativa crítica y transformadora del modelo agroindustrial y que, además, busca integrar acciones surgidas en distintos ámbitos, transformadoras y de cambio social (Gazzano et al., 2021; Navarrete, 2017). Fundada en principios ecológicos y sociales, promueve técnicas específicas y estrategias de organización, participación y acción propias a partir del intercambio de saberes científicos y populares (Sevilla Guzmán, 2011; Altieri, 2007). Si bien el término agroecología fue acuñado alrededor de 1930 por Basil Bensin, agrónomo ruso, recién a partir de 1980 comenzó a difundirse (Wezel y Soldat 2009). Posteriormente, en los '90, Eduardo Sevilla Guzmán y su grupo del Instituto de Sociología y Estudios Campesinos (ISEC) de la Universidad de Córdoba, España, desarrollaron el cuerpo teórico de la sociología agroecológica. En palabras de Gazzano et al:

"la agroecología se vincula con estrategias de resistencia y búsqueda de cambio en el sistema alimentario corporativo, cuestionando y generando opciones ante los efectos negativos biofísicos, sociales, económicos, políticos y culturales en torno a la soberanía alimentaria y más allá de ella, en la definición de las formas de habitar”. (2021, p. 19). 
Wezel et al. (2009) plantean la agroecología como un movimiento social, cultural y político, Guzmán Casado et al. (2000) señalan que involucra las dimensiones ecológico-técnico-productiva, socioeconómico-cultural y sociopolítica. Luego, a partir de esta triple dimensión, Toledo (2011) sostiene que se trata de un mismo proceso que articula tres dimensiones de cambio, tres revoluciones, y a su vez la vincula epistemológicamente con la ecología política, la economía ecológica, la historia ambiental y la etnoecología, como también con los movimientos sociales relacionados con las reivindicaciones de los pequeños productores, campesinos y pueblos indígenas.

La agroecología, superadora de la seguridad alimentaria, persigue la soberanía alimentaria y se enfoca en garantizar el derecho a la alimentación para toda la población, en tanto tiene su eje en el derecho de los pueblos de definir sus estrategias de producción, distribución y consumo de alimentos (Gazzano et al., 2021). Para Toledo (2011), también se orienta hacia la soberanía tecnológica y energética, y en ese sentido, Altieri (1999) hace hincapié en que la agroecología reemplaza el predominio de la tecnología extranjera con tecnologías que se adaptan a condiciones ecológicas locales y específicas, así como a variaciones en el sistema social. Se pone énfasis en el uso de los recursos de producción propia debido a que ayudan a reducir la necesidad de insumos externos. Gliessman, por su parte, define la agroecología como la aplicación de la ciencia ecológica para el estudio, diseño y manejo sustentable de los agroecosistemas. La integración de los diversos sistemas de conocimiento de los distintos actores del sistema alimentario es la propuesta delos movimientos sociales que están promoviendo la transición a sistemas alimentarios soberanos y justos (Gliessman et al., 2019).

Dada la especificidad de cada agroecosistema, para desarrollar tecnologías apropiadas necesita que los productores asuman una posición activa de investigación, no solo de las condiciones agroecológicas locales sino también de las interacciones ecológicas, sociales, económicas y culturales, reconociendo la dependencia de los objetivos de producción del contexto cultural y socioeconómico específico. En consecuencia, no es posible aportar a procesos de transición desde la agricultura tradicional a la agroecológica si se pide al productor que cambie radicalmente el manejo que realiza, menos aún sin conocer al sujeto con quien trabaja (Santos, 2014). Desde esta perspectiva, la agroecología se configura como práctica social, ya que intenta coordinar complejos agroecosistemas insertos en múltiples relaciones naturales y sociales que los determinan y por los cuales son determinados. Siguiendo a Gazzano et al. (2021), la propuesta agroecológica de los movimientos sociales plantea transformaciones en las relaciones sociales y de poder, el acceso y la gestión de los bienes naturales, la distribución de los alimentos y, asimismo, el acceso a ellos, la organización social, la toma de decisiones sobre la orientación del sistema productivo y las formas de creación de conocimiento. Además, integra la dimensión política en su vínculo con las organizaciones sociales y los cambios institucionales necesarios. De esta manera, los movimientos sociales cumplen un rol central en su difusión yadopción en diferentes contextos (Guhur et al., 2017).

El proceso de organización de las comunidades campesinas en Movimientos Sociales (MS) ante el avasallamiento de sus derechos busca generar "espacios de democratización" tanto políticos como culturales, proyectos y emprendimientos económicos y productivos por fuera del sistema de producción agroindustrial (Giarraca y Teubal, 2008). En 2013, Burgos señalaba los casos del Movimiento Campesino de Formosa (MOCAFOR), producto de la ruptura del Movimiento Agrario Formoseño (MAF); el Movimiento Campesino de Córdoba 
(MCC); la Unión de Trabajadores Sin Tierra (UST) de Mendoza; el Movimiento Campesino de Salta (MOCASA); el Movimiento Campesino de Jujuy (MOCAJU); el Movimiento Nacional Campesino Indígena (MNCl), que nuclea a campesinos de siete provincias; el Movimiento Agrario de Misiones (MAM), entre otros. Estos se enmarcan en un contexto de lucha de las comunidades campesinas por recuperar sus tierras y territorios, defender su forma de vida y transformar las relaciones de dominación en el sistema capitalista (Giarraca, 2006). Los diversos movimientos campesinos luchan por crear espacios de comercialización más justos y equitativos, combatir la pobreza, el hambre, el desempleo, y reclaman derechos tales como la soberanía alimentaria, el acceso a la tierra, la salud y la educación, y el respeto por su cultura y saberes (Herrera-Jaramillo et al., 2016). La falta de acceso a los recursos productivos, especialmente a la tierra, es una de las razones clave por las que los campesinos indígenas, que son las poblaciones más afectadas dentro del campesinado, sufren hambre (FIAN, 2003).

El Movimiento Campesino de Santiago del Estero-Vía Campesina (MOCASE-VC) persigue la soberanía alimentaria entendiendo que es el derecho de los pueblos, de sus países o uniones de Estados a definir su política agraria y alimentaria, sin dumping frente a países terceros. El $\mathrm{MNCl}$ la define como el derecho de los pueblos a producir y consumir alimentos sanos que satisfagan las necesidades y se adecuen a la cultura de cada región. En palabras de la Organización de las Naciones Unidas para la Alimentación y la Agricultura (FAO), la definición de soberanía alimentaria es el derecho a proteger y regular la producción nacional agropecuaria y a proteger el mercado doméstico del dumping de excedentes agrícolas y de las importaciones a bajo precio de otros países.

En particular, el $\mathrm{MNCl}$, cuyos objetivos son la reforma agraria integral $\left.\right|^{3}$ y la soberanía alimentaria, se articula con otras organizaciones del campo y de la ciudad; congrega a más de 20000 familias campesinas de todo el país y es el espacio nacional de articulación de las organizaciones campesinas relacionadas con la Vía Campesina (VC). Esta adopta la agroecología como pilar de su accionar hacia la soberanía alimentaria. También se articula con organizaciones de otros países latinoamericanos a través de la Coordinadora Latinoamericana de Organizaciones del Campo-Vía Campesina (CLOC-VC) y forma parte de la Vía Campesina Internacional (Michi 2010).

A este movimiento pertenecen: el MOCASE-VC, el MCC de Córdoba, COTRUM en Misiones, Red Puna, Tierra Fértil en Jujuy, Servicio a la Cultura Popular (SERCUPO) en Buenos Aires, Mesa Campesina del Norte Neuquino (MCNN) en Neuquén, UST en Mendoza y San Juan, y Encuentro Calchaquí en Salta (Vidal, 2015).

En cuanto a la construcción de la soberanía alimentaria y la conexión de esta con la agroecología, para todo el $\mathrm{MNCl}$ y la $\mathrm{VC}$ es extremadamente importante fortalecer la autodeterminación de los pueblos y volver al modo de producción ancestral. Por eso el modelo pedagógico que se plantea desde el MOCASE en la UNICAM-SURI se basa en la educación popular como forma no hegemónica de construcción de conocimientos para la subsistencia del campesinado y fomento de la producción de alimentos sanos. El objetivo de este artículo es reflexionar sobre los aportes que genera el proyecto pedagógico de educación popular de la UNICAM-SURI al MOCASE y a todo el movimiento indígena-campesino, además de

3) Reforma agraria en la cual el acceso a la tierra debe estar acompañado por políticas y programas de apoyo a la agricultura campesina. 
analizar cómo esta estrategia de educación contribuye a la construcción de la soberanía alimentaria al fomentar la agroecología. ${ }^{4}$

Para alcanzar este objetivo, el artículo está estructurado en cuatro apartados. El primero de ellos se refiere a la visión de la extensión universitaria que enmarca la experiencia y su consecuente abordaje metodológico; el segundo intenta plantear de manera general las características salientes de los proyectos pedagógicos de los movimientos sociales; el tercero describe el modo en que se constituye la universidad campesina, la primera del MOCASE con trayecto pedagógico; y en el cuarto apartado se analiza cómo realiza sus aportes al movimiento indígena-campesino y a la soberanía alimentaria.

\section{La visión de la extensión universitaria que subyace en el abordaje metodológico de la experiencia}

Si bien los modelos de extensión universitaria asumen su función social, comparten su cultura y conocimientos con personas, grupos o comunidades marginados o vulnerables, López (2020) diferencia dos lógicas de extensión universitaria, la llamada extensionista y la vinculacionista. En el caso de la extensionista, no economicista, puede presentarse desde las perspectivas asistencialistas y crítico-emancipatorias (López, 2020). El extensionismo con enfoque crítico-emancipatorio considera la extensión universitaria como una herramienta para la superación de la desigualdad. Dado que es propio de sociedades desiguales, también lo es de la extensión universitaria latinoamericana (López, 2020), por ello se enmarca, desde la década del 70 , en la necesidad de un giro descolonial debido a la crisis epistémica del modelo educativo mundial eurocéntrico consolidado con la colonización, el neoliberalismo y el capitalismo global hasta la actualidad (Macedo, 2020). Los principales representantes del extensionismo crítico-emancipatorio son Paulo Freire en Brasil (1973) y Augusto Salazar Bondy en Perú (1973). Dentro de este enfoque, se destacan los estudios referidos a la extensión crítica trabajada en Uruguay por Tommasino y Cano (2016); en Argentina, la integración social (Arrillaga y Marioni, 2015);en México, la concientización (Serna Alcántara, 2007); y por último, en Cuba, el desarrollo integral (González González y González Fernández Larrea, 2003). Esta mirada de la extensión universitaria tiene por objetivo el mejoramiento de la vida de los involucrados y del tejido social de la zona de influencia, pero no se basa en la acumulación económica como instrumento para tal fin. En esta concepción la demanda surge de la interacción social entre el sujeto extensionista y el sujeto destinatario porque ambos participan de la definición de los objetivos y los contenidos de las acciones (López Geronazzo y López, 2018). Por ello la metodología propuesta por los proyectos de extensión dentro de esta concepción se inscribe en las formas de investigación aplicada y en la investigación participativa. Se apoyan en la Investigación-Acción Participativa (IAP), que reconoce un papel fundamental en el proceso de indagación y en las decisiones relativas a sus proyectos

4) Este trabajo ha sido financiado por el proyecto denominado "Transformaciones en la estructura agraria y uso relativo de los factores directos de producción. El factor tierra en las provincias de Buenos Aires y Santiago del Estero". Secretaría de Ciencia y Técnica. Universidad de Buenos Aires. Programación Científica 2020-2021. Código: 20020190200088 BA y el proyecto UBANEX 2017 denominado "Desarrollo e incorporación de tecnologías apropiadas para el fortalecimiento de la producción agroecológica en la UNICAM-SURI, en búsqueda de la soberanía alimentaria". 
políticos, sociales y productivos de los propios colectivos estudiados. La IAP se sustenta en tres cuestiones: la imprescindible relación acción-reflexión y teoría-práctica, el compromiso de los investigadores con las organizaciones y movimientos populares, y el lugar protagónico en todo el proceso de investigación de quienes habitualmente son concebidos como objetos, lo que suele sintetizarse como transformación del objeto en sujeto (Rigal, 1997).

En este artículo analizamos el trabajo de campo realizado en el marco de un proyecto de extensión universitaria de la Universidad de Buenos Aires (UBANEX) diseñado para dar respuesta a las necesidades planteadas desde las organizaciones a través de estudiantes y graduados de la Facultad de Agronomía de la Universidad de Buenos Aires (FAUBA) que participan y apoyan las actividades del $\mathrm{MNCl}$. El proyecto denominado "Desarrollo e incorporación de tecnologías apropiadas para el fortalecimiento de la producción agroecológica en la UNICAM-SURI, en búsqueda de la soberanía alimentaria", del Programa UBANEX 2017, se desarrolló durante 2018 y parte de 2019. Se implementó como Práctica Preprofesional de la FAUBA mediante las "Pasantías vivenciales" de verano e invierno a distintas provincias para conocer las comunidades campesinas del $\mathrm{MNCl}$, sus proyectos productivos y sus cooperativas, de modo de desarrollar en los estudiantes la observación participante.

Para recopilar información de primera mano se hizo el trabajo de campo en el predio donde se lleva adelante el proyecto de la UNICAM-SURI. Durante ese trabajo de campo se intentó mantener un equilibrio entre la observación y la participación. Se presenciaron clases, actividades grupales y charlas, se tomaron notas y fotografías, también se grabaron entrevistas. Y luego de un proceso de discusión colectiva con todos los integrantes de la UNICAM-SURI y sus organizaciones se consensuó cómo y qué relevar para generar la información necesaria para generar tecnologías apropiadas. Fundamentalmente, se propusieron dos procesos complementarios a partir de lo consensuado, uno de ellos fue la observación directa y sistematización, y el otro, el diálogo con técnicos, campesinos, campesinas y referentes del movimiento acerca de los aportes del proyecto pedagógico al movimiento indígena campesino y a la soberanía alimentaria. En este proceso se prestó especial atención a los saberes puestos en juego por la comunidad involucrada en el proceso pedagógico de la UNICAM-SURI así como a los producidos a partir del trabajo conjunto.

Retomando el objetivo de este artículo, para alcanzarlo, la metodología se dividió en dos etapas. En una primera etapa se analizaron los antecedentes en Argentina sobre los proyectos pedagógicos de los movimientos sociales, se realizó una revisión bibliográfica que abordó la historia de la creación de la UNICAM- SURI e información sobre el contexto socioeconómico productivo en la zona de influencia de la universidad. En una segunda etapa se analizó la información recopilada en el proyecto UBANEX desde la perspectiva del análisis y la reflexión sobre los aportes que genera el proyecto pedagógico de educación popular de la UNICAM-SURI al MOCASE y a todo el movimiento indígena-campesino y, a través del fomento de la agroecología, su contribución a la soberanía alimentaria.

\section{Los proyectos pedagógicos de los movimientos sociales}

Durante las últimas tres décadas, en Latinoamérica se crearon universidades y otras Instituciones de Educación Superior (IES) orientadas a la inclusión de indígenas y campesinos al estudio de licenciaturas o diplomaturas. Según Mato (2015), estas modalidades educativas 
inclusivas, denominadas indígenas y/o interculturales, han sido promovidas en algunos casos por organizaciones $y / o$ dirigentes indígenas $y$, en otros, por universidades e IES "convencionales" mediante alianzas entre ambos tipos de instituciones o por iniciativas de los Estados.

Estas iniciativas son más recientes en Argentina que en algunos países de la región. En ese sentido, Baraldo (2010) destaca el impulso que tomó a partir de la reflexión conjunta entre investigadores, docentes de escuelas públicas estatales, miembros de movimientos sociales y educadores populares de diversos países en el Foro Mundial de Educación, que tuvo lugar en Buenos Aires en mayo de 2006. Sobretodo señala la importancia de los encuentros de la mesa de trabajo denominada "Teoría e Historia de la Educación Popular", que tomó entre sus ejes a los movimientos sociales y sus experiencias educativas en Argentina y otros países. En 2007 se creó el Área de Formación Cogestionada con los Movimientos Sociales dentro del Observatorio Social de América Latina (OSAL) del Consejo Latinoamericano de Ciencias Sociales (CLACSO) y del Centro de Investigación y Formación de Movimientos Sociales Latinoamericanos (CIFMSL), ambos bajo la coordinación de la periodista, educadora popular y feminista Claudia Korol.

Surge entonces la necesidad de encarar la construcción colectiva de conocimientos mediante al articulación de saberes académicos y populares con el objetivo de fortalecer procesos de emancipación social como un gran desafío (Baraldo, 2010). Según Burgos (2013), la dimensión político-pedagógica, que es una de las más importantes, constituye uno de los aspectos menos analizados de los movimientos sociales de reciente desarrollo, puesto que los nuevos impulsan la construcción de una forma de participación horizontal con la mirada puesta en la transformación de la sociedad capitalista. Así, para superar la subordinación del trabajo al capital, proponen sistemas alternativos de producción y comercialización y enfocan la formación de sus integrantes hacia la disputa con los modelos económicos hegemónicos (Zibechi, 2005; Korol, 2007; y Michi, 2010). Por otra parte, Burgos (2013) sostiene que los nuevos movimientos sociales dan cuenta de la emergencia de formas de acción colectiva que no son homogéneas ni estáticas como las de los "viejos" movimientos sociales de principios del siglo XX; ya que no reducen la lucha social a la dicotomía capital-trabajo.

Dado que la educación es un aspecto esencial, según Zibechi (2003), una característica de los nuevos movimientos sociales es su capacidad para formar intelectuales propios tomando en sus manos la educación y la formación de sus dirigentes. Sin embargo, la mayoría de las investigaciones que abordan su accionar en términos educativos profundiza las estrategias desarrolladas como respuesta al desempleo y la pobreza (Baraldo, 2009). La formación para el trabajo en los movimientos sociales es política porque su objetivo es romper con la subordinación del obrero al capital y, por lo tanto, no se orienta a la inserción de los individuos en el mercado de trabajo como asalariados. Guelman (2012) estudia la relación educación-trabajo en la capacitación laboral en empresas recuperadas integradas o articuladas con movimientos sociales y la compara con la de las empresas que buscan formar para la flexibilidad, las competencias y la empleabilidad. Palomino (2003 y 2004) aborda la relación educación-trabajo en los movimientos sociales para alcanzar el desarrollo de emprendimientos autogestionados y señala que se diferencian del modelo capitalista porque intentan construir un espacio público donde el trabajo no se intercambia solamente por una retribución monetaria. Para Palomino, el trabajo fomenta la creación de lazos sociales en la base de la organización de los movimientos sociales, constituyéndose como una dimensión 
política. La educación popular siempre es una posición política, político-pedagógica y no una modalidad educativa, es una práctica pedagógica descolonizadora ${ }^{5}$ que valoriza y recupera experiencias y saberes negados y silenciados cuando los problematiza y los pone en diálogo y tensión con saberes legitimados (Guelman, 2014).

\section{Santiago del Estero y la UNICAM-SURI como proyecto pedagógico del MNCI}

Santiago del Estero se encuentra ubicada dentro del GCA y cerca del $40 \%$ de la población total de la provincia es rural, con campesinos que, en parte, habitan en tierras fiscales donde, una vez agotada definitivamente la actividad forestal, las grandes empresas se retiraron y quedaron los extrabajadores y sus familias ocupándolas (Guaglianone, 2001). Según documentos de la Mesa de Tierras de Santiago del Estero, ${ }^{6}$ en el campo vivirían alrededor de 28000 familias, por ello tiene uno de los índices de ruralidad más altos del país, con un $33,9 \%$, cuando este indicador para el total de Argentina es del 10,7 \%. Muchas de estas familias son poseedoras con ánimo de dueño de las tierras que durante varias generaciones han ocupado pero no tienen regularizada su situación dominial. Según el defensor del pueblo de la provincia, en 2003 había alrededor de 24000 poseedores legítimos expuestos a ser desalojados (Ministerio de Justicia, Seguridad y Derechos Humanos, 2003). Las poblaciones originarias que habitan la provincia son vilela, diaguita, lule vilela, sanavirón, cacano, guaycurú y tonocoté o zurita (Vidal, 2015). Los departamentos que tienen más población en situación de pobreza son además los que concentran la mayor cantidad de población rural.

Las familias rurales santiagueñas tienen como sustento principal la producción ganadera extensiva de caprinos dentro de una producción animal diversificada (también cerdos, gallinas, vacas, ovejas) y otras actividades como la extracción de leña para consumo familiar, producción de carbón y siembra de cultivos como maíz, zapallo, sandía. Estas actividades son determinadas en gran parte por el clima cálido correspondiente a regiones subtropicales y por la escasez de agua y de redes de agua corriente, tanto para consumo familiar como para las diversas producciones. En el marco de una provincia con una importante población campesina e indígena, altamente empobrecida, con carencia de infraestructura y con una mínima experiencia de organización, surgió, el 4 de agosto 1990, hace más de 30 años, el MOCASE. Este movimiento social en 2001 sufrió una escisión y quedó dividido en dos organizaciones: MOCASE y MOCASE-VC. Según Michi (2010), del MOCASE-VC forma parte todo el campesinado, y señala que con el tiempo se fue consolidando en sus tres dimensiones actuales: la lucha por la resistencia por el territorio, los espacios de autogobierno y autogestión, y el movimiento como actor político provincial, nacional e internacional. A nivel nacional forma parte del $\mathrm{MNCl}$, el cual nuclea organizaciones de base del monte profundo del país y lleva líneas de trabajo en defensa de los derechos humanos: derecho al territorio, a una vivienda digna, a una alimentación sana, a la educación, al agua, y al respeto de sus culturas. En ese contexto, el MOCASE-VC es una organización campesino-indígena com-

5) Argumedo (1996) define a la pedagogía descolonizadora como aquella comprometida con la identidad de los pueblos latinoamericanos desde un proyecto político emancipador.

6) Desde el año 2000, está integrada por organizaciones campesinas, la Pastoral Social de la Iglesia Católica y diversas ONG (INCUPO, Be Pe, El Ceibal, PRADE, FUNDAPAZ, entre otras). 
puesta por más de 12000 familias que se movilizan por la producción de alimentos sanos, por la agroecología, la justicia y el cambio social (MOCASE, 2017).

Este movimiento social tiene tres instancias de organización y participación, las "comunidades de base", las "centrales" y los "secretariados". Las primeras son agrupaciones de 30 o 40 familias cercanas geográficamente (entre 100 y 200 kilómetros) que se reúnen en asambleas para coordinar la producción y garantizar la soberanía alimentaria sobre la base de prácticas que respetan el equilibrio ecológico y social en oposición a una forma de producción basada en una utilización intensiva de los recursos naturales y orientados por racionalizaciones, para discutir sus problemáticas, desalojos ilegales de sus tierras, la contaminación de agua, la tala indiscriminada de árboles, la destrucción de la tierra, y para elegir los representantes y los temas principales a ser profundizados en las organizaciones de segundo grado, que son las "centrales". Articulado en 10 departamentos de la provincia que conforman 5 centrales, adoptó como principios fundamentales la lucha por la defensa de la tenencia de la tierra, los derechos de los campesinos en materia de educación, salud, trabajo y alimentación, y se constituyó como actor político a nivel provincial, nacional e internacional (Burgos, 2017). En las centrales participan en asamblea todos los representantes de las comunidades de base de una misma región. Por último, el tercer nivel de organización se compone de 5 "secretariados": Secretaría de Salud; de Tierra, Derechos Humanos y Medio Ambiente; de Comunicación; de Producción y Comercialización; y de Formación, y en ellos participan los representantes de las diferentes centrales y se determinan las principales líneas de acción del movimiento en lo productivo, político y educativo.

Desde sus comienzos, el MOCASE asumió como estrategia central la lucha por la tenencia de la tierra y por las condiciones de vida de las familias campesinas indígenas. Esto ha implicado una difícil relación con el sistema político y sus prácticas más tradicionales. A pesar de ello, el MOCASE ha conformado una organización autónoma, con iniciativas de botiquines comunitarios, construcción de centrales y casas campesinas, módulos de acopio y extracción de agua, creación de agroindustrias, carnicerías comunitarias instaladas en los pueblos de referencia, recreación de tecnologías indígenas, entre otras. El MOCASE-VC ha desarrollado en Santiago del Estero dos proyectos pedagógicos: la Escuela Agroecológica y la UNICAM-SURI, ambos de educación popular desde una perspectiva Latino Americana de la pedagogía descolonial. En palabras de Macedo:

"el sujeto 'indocampesino' es portador de su propia voz, sujeto-actor colectivo e histórico que emprende una lucha horizontal, para la autonomía y la liberación de los pueblos de la lógica capitalista hegemónica, la modernidad-colonialidad y el patriarcado causador de epistemicidio sistémico en Latinoamérica". (2020, p. 13)

La idea de pensar en una universidad campesina venía gestándose desde tiempo atrás. En el año 2006, estudiantes de las Universidades de Buenos Aires y de Córdoba visitaron Santiago del Estero por unas pasantías y comenzaron a encuestar a los jóvenes para ver qué querían estudiar. De esas encuestas surgieron cosas interesantes, como el deseo de esos jóvenes ser policía, chef, maestro o médico. Es así como se comenzaron a poner en evidencia las demandas de la juventud y el hecho de que muchos no terminaban la escuela secundaria. En 2007, por iniciativa de niños, jóvenes y padres, nació la Escuela de Agroecología del MOCASE y comenzó a hacerse más fuerte la idea de crear una universidad 
campesina. En ese contexto, para la construcción popular de conocimiento surgieron los espacios de formación de promotores de salud, los comunicadores populares, los promotores productivos y luego de la UNICAM-SURI. Esta Universidad fue inaugurada por el $\mathrm{MNCl}$ el 17 de abril de 2013, el "Día Internacional de la Lucha Campesina", en la localidad de Villa Ojo de Agua, departamento del mismo nombre, debido a la necesidad de los y las integrantes del Movimiento de generar un espacio para los encuentros, construir herramientas de análisis y transformar la realidad en los territorios y las comunidades.

Según datos del Censo 2010, en este departamento hay 15354 habitantes, su superficie es de $6269 \mathrm{~km}^{2}$, limita al norte con los departamentos de Loreto y Atamisqui, al este con el departamento Quebrachos, al oeste con el departamento Choya, y al sur con la provincia de Córdoba. La localidad Villa Ojo de Agua, fundada en 1911, es la ciudad cabecera del departamento, se encuentra a $209 \mathrm{~km}$ de la capital provincial, en una zona serrano minera del sur de Santiago del Estero, atravesada por la Ruta Nacional № 9 . Tiene la particularidad de estar asentada sobre una olla natural que absorbe el agua de lluvia y la almacena para que luego sea utilizada para el consumo de la población mediante perforaciones. En el pasado, cuando las lluvias eran abundantes se desbordaba la olla y se veían brotar vertientes naturales que los nativos llamaban Ojo de Agua. Las 12 hectáreas de la Universidad se hallan a cuatro kilómetros de esta localidad, en tierra cedida por un chacarero de la zona. Las edificaciones de su predio, construidas por jóvenes de las organizaciones y de algunas comunidades indígenas de Santiago que viven y se forman allí, se utilizan para dictar las clases, cuentan con una sala de administración y otra para los coordinadores y un salón para dormir o desarrollar las asambleas. Muchas de las comunidades que se acercan a la UNICAM tienen conflictos de tierras y encuentran que, además de ser una Universidad campesina, es una central más de la organización que les brinda apoyo para contener esos conflictos.

"La UNICAM-SURI tiene convenios con otras universidades pero formalmente tiene la personería jurídica de una comunidad indígena vecina, del pueblo sanavirón, funciona, además de como universidad, como central del MOCASE". (Entrevista a técnica, 14 de marzo de 2019)

Es un espacio de formación "pensado desde el pueblo" donde la comunidad es la protagonista. De acuerdo con la página web del MOCASE:

"La UNICAM es un espacio donde campesinos, indígenas, trabajadores urbanos y rurales ejercen el derecho a una formación que les permita no solo recuperar y reivindicar su propia cultura, sino también trabajar para educar desde otra mirada".(MOCASE, 2017)

En la UNICAM, la educación popular emplea metodologías pedagógicas y didácticas basadas en la complementación de distintos saberes orientados a cambiar cuestiones sociales a través de la praxis, la participación y el diálogo. Nació con la intención de promover la formación de los jóvenes en carreras necesarias para la recreación de la vida campesina y para el desarrollo del movimiento, intentando evitar la migración en busca de trabajos urbanos así como los riesgos de fracaso de las experiencias universitarias urbanas (Michi, 2010). Es importante señalar que, según Torres Carrillo (2008), puesto que es preciso generar conocimiento sobre las realidades y saberes construidos en las instancias de formación, sobre 
los sujetos y sobre los contextos donde se desenvolvían, el proceso de registrar y el de sistematizar forman parte de la metodología de la educación popular como también de la IAP.

El estudio en Movimientos Campesinos y Educación realizado en 2010 por Michi sintetiza en dos los objetivos fundamentales de la Universidad Campesina: el primero es formar sus propios técnicos para trabajar en las zonas rurales, y el segundo, brindar oportunidades a los jóvenes para "estudiar" y "formarse más sistemáticamente", ya que el índice de superación de los primeros niveles de educación formal primaria, secundaria y universitaria, son muy bajos en la provincia. Esta Universidad surgió de la necesidad de que los hijos y las hijas de la población campesina y de la población en riesgo de exclusión de las zonas urbanas puedan optar por cursar estudios superiores bajo la óptica campesina. En agosto de 2013, en la UNICAM-SURI se formaban cerca de 30 jóvenes de origen campesino y urbano de diferentes provincias del país acompañados por 6 personas del equipo pedagógico y de coordinación.

La UNICAM se desarrolló de manera conjunta con las Universidades Nacionales de Quilmes (UNQ) y de La Plata (UNLP) a partir de diversos procesos educativos realizados en diferentes instancias del $\mathrm{MNCl}$, como las Escuelas Campesinas de Agroecología de Santiago del Estero, Córdoba y Mendoza, las Escuelas de la Memoria Histórica, los Campamentos Latinoamericanos de Jóvenes y las Escuelas de Formación Política. La currícula ofrece ocho itinerarios o trayectos pedagógicos: Agroecología, Comunicación Popular, Derechos Humanos, Tierra y Territorio, Artes y Música Populares, Medicina Popular, Arquitectura Popular, Tecnologías e Industrias Renovables, Economía Popular y Solidaria. La idea de la organización es poder replicar esta experiencia pedagógica en otras provincias para que cada proyecto pueda enfocarse más hacia las necesidades y saberes zonales. Los cursos se desarrollan en tres años y se obtiene una diplomatura. En la actualidad, la oficialidad de los estudios está garantizada por un convenio con la UNQ y la UNLP. Este convenio tiene el apoyo del Ministerio de Cultura y del Ministerio de Educación de la Nación, de la Subsecretaría de Agricultura Familiar, y de varias organizaciones del campo popular (Mato, 2015).

Tanto los docentes como los estudiantes universitarios que formaron parte del proceso de consolidación de la Universidad Campesina modificaron su habitual método de enseñanza-aprendizaje eurocéntrico para formar parte del proyecto de educación popular propuesto por el MOCASE-VC. La educación popular plantea dar forma a un paradigma latinoamericano con especificidades identitarias, históricas, de contexto y de luchas. En ese sentido, América Latina ha desarrollado conceptos y prácticas críticas a la forma de la cultura y la colonialidad mediante el diálogo, la confrontación de saberes y la negociación cultural como eje de su propuesta metodológica (Mejía, 2014). Para alcanzar la negociación cultural y el diálogo de saberes es imprescindible, previamente, reconocer una cultura como propia, por ello en la UNICAM-SURI, los jóvenes del campo y la ciudad de diferentes puntos del país transitan una experiencia educativa que valoriza sus saberes ancestrales, comunitarios, sin sufrir desarraigo de sus distintos territorios, lo cual les posibilita ser parte de un sistema educativo que integra a las clases sociales más humildes. Los contenidos de las asignaturas cuestionan la cultura eurocéntrica y la pedagogía escolar tradicional. El modo de organización de la educación popular se propone democratizar las relaciones sociales y distribuir el poder (Gluz, 2011). El énfasis está puesto en la cultura oral y no necesariamente en la escritura, ya que no todos los que estudian saben leer y escribir. La idea es rescatar esa otra forma de aprender y de enseñar a través de la palabra, de estar en ronda y de escucharse, sin desconocer la im- 
portancia de aprender a escribir y hacer memoria escrita. Los estudiantes expresan su propio pensamiento y son escuchados como sujetos políticos (Gluz, 2010). En esta otra forma de aprender y de enseñar los adultos mayores tienen más para aportar que los jóvenes, transmiten sus saberes ancestrales y toman así un rol preponderante. El trabajo diario es intenso, dictado de clases, talleres y muchas tareas grupales. En ese sentido, los estudiantes sistematizan experiencias productivas con orientaciones agroecológicas ancestrales. Este proceso, fundamento de la educación popular, busca diferenciarse de las formas hegemónicas de producción agropecuaria, que utilizan gran cantidad de insumos químicos y semillas modificadas genéticamente, y en cambio toma las prácticas ancestrales de cultivo donde el hombre es parte de la naturaleza y el control de malezas y otros patógenos se realiza con bioinsumos y se siembran semillas nativas que mantienen la variabilidad genética, conservan la poza génica y con ello la biodiversidad. Como contrapartida, la productividad con frecuencia se ve afectada por las condiciones agroecológicas locales -temperaturas extremas y escasez de aguay sociales - falta de acceso al agua de red, sistemas de salud y herramientas técnicas.

\section{Aportes de la UNICAM-SURI al movimiento indígena campesino y a la soberanía alimentaria}

En Argentina, así como en el resto de América Latina, sumada a la ausencia de políticas que intenten conservar el contenido social del medio rural, la tendencia neodesarrollista impulsa la producción de cultivos destinados a la exportación y no la de alimentos para la población local y la soberanía alimentaria, situación que sumiría en la inviabilidad a los pequeños productores (Segrelles Serrano, 2010). El MOCASE-VC plantea transformar el sistema social y tecnológico de producción y comercialización de alimentos integrando el derecho a la tierra y al agua de sus habitantes (Juárez et al., 2016). Sus aportes al campo de las Tecnologías Inclusivas Sociales (TIS) han sido analizados por el equipo de investigación del Área de Estudios Sociales sobre la Tecnología y la Innovación (AESTI) de la UNQ, se han realizado investigaciones sobre la estrategia global del Movimiento de La Vía Campesina (y del MOCASE en Argentina) en términos de innovación social transformadora y sistémica asociada a la perspectiva agroecológica (Juárez et al., 2018).

EI MOCASE-VC facilita que los campesinos, junto con el resto de estudiantes de las otras comunidades, puedan acudir a la Escuela de Agroecología de Quimilí, creada en 2007 y cuyo proyecto pedagógico podría tratarse en otro artículo debido a su larga trayectoria. El proyecto universitario campesino busca además articular con diferentes instituciones académicas con el objetivo de consolidar los trayectos pedagógicos para contribuir al fortalecimiento del rol del campesinado y a la consolidación de la agroecología y el desarrollo rural sostenible e inclusivo. A este respecto, la UNICAM propone fomentar la incorporación y el desarrollo de tecnologías inclusivas sociales para la producción agroecológica que aporten al desarrollo territorial de su zona de influencia yal proyecto de soberanía alimentaria que persigue la organización. En esos ámbitos de formación plantean debatir cómo utilizar prácticas y tecnologías vinculadas a la agroecología para transformar el sistema agropecuario y agroalimentario concentrado y exportador argentino. La agroecología es vista por el MOCASE-VC como una estrategia sistémica sociotécnica para generar inclusión campesina, soberanía alimentaria y sustentabilidad de los territorios (Juárez, 2016). 
La UNICAM-SURI cuenta con espacios de producción animal y producción vegetal, los cuales sirven también para el autosustento e intercambio con las comunidades campesinas cercanas. Asimismo, poseen un espacio dedicado al cuidado y proceso pedagógico de los niños y niñas, una radio comunitaria llamada FM Suri Manta 89.9, un espacio de cocina, otro de construcción dedicado a completar las obras edilicias -aljibes, aulas y dormitorios, etc.- y un espacio propio destinado a la salud para poder hacer frente a las demandas del cuidado revalorizando sus prácticas ancestrales.

"Es como un complemento del pueblo y también es tener nuestro propio lugar para que contemple las cosas que nosotros pensamos que son también salud; además se hacen talleres de salud y se llevan a las comunidades”. (Entrevista a técnica, 14 de marzo de 2019)

Las primeras camadas de estudiantes fueron de campesinos con conocimientos de agroecología; sin embargo, actualmente, también participan del proyecto estudiantes urbanos de Añatuya, de barrios humildes de Santiago del Estero capital y de Buenos Aires. Este cambio sucedió al tomar la iniciativa de armar un módulo productivo y demostrativo para adolescentes, jóvenes y adultos referentes con consumos problemáticos, pensando en que la participación activa en el proyecto, además de generar un espacio de aprendizaje que transforme su realidad cotidiana, construye lazos de identificación y sentimientos de pertenencia.

"Para que tengan un espacio de trabajo permanente, porque si algo que creemos acá es que el trabajo te encamina, te genera un proyecto, por ahí una cotidianeidad con los chicos, eso de dejar de pensar, como que el trabajo ordena mucho la dinámica, por eso también la estructura que tenemos desde el nivel organizativo, hay muchos compañeros que se han rescatado. Y los que se han rescatado después se convierten en militantes de su organización, en referentes en sus áreas de trabajo, y que después, cuando vuelven a sus comunidades, empiezan a participar mucho más fuerte en lo organizativo y productivo". (Entrevista a referente del movimiento, 13 de marzo de 2019)

La modalidad de asistencia consiste en un sistema de alternancia por el cual los estudiantes permanecen en el establecimiento siete días cada dos o tres meses, lo que les permite aplicar los conocimientos adquiridos en sus territorios de procedencia.

"Para no desarraigar a los jóvenes del medio rural o de los territorios, también para que no dejen de hacer las cosas que tienen que hacer, se organiza una semana al mes o una semana cada dos o tres meses y de sol a sol el trabajo, y con tareas que se llevan para hacer en los territorios, para poner en práctica en parte y para desarrollar ahí". (Entrevista a técnico, 14 de marzo de 2019)

La UNICAM no solo funciona para los campesinos y las campesinas, sino también para todas las personas que quieran aprender otra forma de hacer, otra forma de construir. Se trata de un modo novedoso de enseñanza, como una aproximación de educación popular distinta a la de la academia formal:

"Acá pasa gente de Misiones, santiagueños, salteños, porteños, santafesinos, hay de todos lados. Es también el enfoque metodológico, la forma de trabajar, nosotros sabemos que la UNICAM sola es solo un arroyito en un río que debería ser. La idea es replicar la 
experiencia en otros lugares, en otras provincias, que haya montones de lugares como este, que trabajen más en función de la zona". (Entrevista a técnico, 14 de marzo de 2019)

A nivel formal no funciona como otras universidades, sino más bien como un bachillerato: "Porque tiene otra libertad para impartir los contenidos y no relegamos la cuestión organizativa. Después que cada año es distinto, (...) si un año vienen chicos de una zona en particular se trata de repensar los contenidos para que tengan que ver con su realidad". (Entrevista a técnico, 14 de marzo de 2019).

Además de los cursos, del trabajo de campo y de coordinación y gestión en equipo dentro de la UNICAM, las tareas se llevan a cabo junto con las comunidades: "nosotros tratamos de promover algunas prácticas, como cuestiones productivas en comunidades" (técnico).

En el predio de la UNICAM no se utilizan agroquímicos, solo biofertilizantes de producción propia que aplican con mochilas. Está organizado en pequeños módulos productivos, de porcinos, de vegetales (vivero y producciones agroecológicas de ají, aromáticas y medicinales, papines, batatas, topinambur, zapallo, sandías) y de semillas. No cuentan con un módulo de apicultura porque las abejas se ven afectadas por la falta de agua.

La denominada "casa de las semillas" es una construcción de adobe donde se almacenan semillas de una temporada a otra pero con la lógica de llevarlas a las comunidades e ir reproduciendo variedades adaptadas a la zona y como forma de resguardar y multiplicar la semilla nativa. Guardan y reproducen semillas de intercambio de distintos lugares del país y Latinoamérica. Tienen tanto especies de distintas temporadas, variedades específicas para la zona, medicinales, así como semillas de árboles diversos. Estas semillas se utilizan para hacer experimentos, para llevar a las comunidades, para que se recupere el hábito de la agricultura, de conservarlas, y para distribuirlas a quien necesite para poder producir.

"La lógica es ir reproduciendo más que nada, hacer llegar a las comunidades, este año estuvimos haciendo un trabajo fuerte de entregar semillas para las comunidades y van re bien esas semillas. Hay muchas variedades de maíz, este año probamos cinco pero tenemos un montón. La idea es que sirvan para hacer pruebas acá y poder ir reproduciendo la semilla y también para ver si funciona, si se aguanta más la sequía". (Entrevista a técnica, 14 de marzo de 2019)

"De hecho, muchas comunidades que se llevaron semillas llegaron a la UNICAM por conflictos de tierra, y cuando le mostrábamos la UNICAM, que además de ser una universidad campesina es una central más de la organización que tiene esa función de poder contener a las comunidades con esos conflictos". (Entrevista a técnica, 14 de marzo de 2019)

"Con todo lo que es el desmonte y el cambio climático a nivel local se re siente. Comunidades que antes sembraban pero ahora hace 11 años que no por la seca, porque cambió el patrón de lluvias, el año pasado también hicimos experiencia con algunas comunidades más cerca de Quimilí en Añatuya, han llevado semillas de acá con esa idea de autoproducción, pero también de multiplicar la semilla criolla". (Entrevista a técnica, 14 de marzo de 2019) 
El alcance que tiene la UNICAM en cuanto al resguardo y a la recirculación de las semillas de producción propia es incuantificable, ya que tanto las personas que cursan en la Universidad como en los talleres o las visitas que hacen en escuelas o dentro de las comunidades, o en actividades llevadas a cabo por la CLOC o la Vía Campesina las semillas y la experiencia de la UNICAM se "esparce".

"A todas las escuelas que fuimos, todas tienen chacra o cercos hechos con las semillas de la UNICAM. Cuando se estaba tratando la Ley de Semillas, en las movidas que se estaban haciendo en Congreso con intercambio de semillas, las semillas que se estaban repartiendo, un montón eran de la UNICAM. Además esto es parte de la estrategia latinoamericana, hay también otros institutos en otros países con los que se intercambian semillas. El alcance es a veces incuantificable". (Entrevista a técnico, 14 de marzo de 2019)

"Guardar la semilla da autonomía y es pensar la agricultura como forma de vida y no como simple producción”. (Entrevista a técnica, 14 de marzo de 2019)

A pesar de que poseen un registro de las familias que se llevan las semillas que reproduce la UNICAM, no es posible cuantificar qué incidencia tiene en la producción Además, los productores que se llevan semillas de la UNICAM luego aportan otras para guardar para el año siguiente. Además de promover el uso de la semilla propia -autoprovisión de la semillas-, también fomentan el no uso de agroquímicos puesto que hacen ellos mismos sus preparados, lo que, por una parte, alimenta la autonomía de las comunidades y, por otra, promueve la agroecología como modo de vida.

"Nosotros vemos que a partir de las prácticas agroecológicas mejoramos pero no lo tenemos cuantificado. Siempre nos dicen los productores que las semillas que compran no les sirven y que las de acá son buenísimas y todos dicen lo mismo, y no tenemos cuantificado eso, sí tenemos fotos de cuando vamos a las comunidades, o zapallos que nos dan para que guardemos las semillas, nosotros les pedimos que nos den un puñado de semillas de las que se llevan porque también es nuestra tarea resguardar las semillas". (Entrevista a campesino, 13 de marzo de 2019)

En 2018, la Universidad Campesina trabajó con técnicos del Instituto Nacional de Tecnología Agropecuaria (INTA) de Salta en la recuperación de maíces nativos, se probaron cinco variedades con la idea de reproducirlas para que las pudieran utilizar las comunidades locales debido a su mayor resistencia a la sequía.

A modo de síntesis, las demandas sociales y ecológicas emanadas desde el pensamiento crítico abordadas desde el proyecto pedagógico de la UNICAM-SURI son: la conservación del suelo y del agua; el mantenimiento de la biodiversidad; la producción y utilización de bioinsumos; el no uso de transgénicos; la autogestión y autosuficiencia locales; la soberanía alimentaria y los alimentos sanos; los mercados justos y populares; la equidad agraria; el diálogo de saberes y la IAP. 


\section{Reflexiones finales}

En la Argentina, los proyectos pedagógicos de los movimientos sociales son recientes. Sin embargo, el MOCASE-VC trabaja en ello desde hace más de quince años. La UNICAMSURI promueve la transformación del concentrado sector agropecuario argentino desde un proyecto pedagógico focalizado en los actores más desfavorecidos en el sistema hegemónico actual. Su propuesta pedagógica busca crear espacios de discusión y formación sobre soberanía alimentaria, agroecología, acceso a la tierra, salud, educación, respeto por la cultura y los saberes. El paradigma pedagógico de la UNICAM-SURI articula e integra a los pequeños productores campesinos con la comunidad académica y los movimientos sociales con la intención de provocar transformaciones contrahegemónicas sustanciales en el sistema agroalimentario. Para desarrollar su propuesta pedagógica, en un contexto de lucha de las comunidades campesinas por recuperar sus tierras y territorios y defender su forma de vida, enfrenta grandes desafíos que podrían superarse mediante la mayor articulación con distintas instituciones gubernamentales y universidades que adhieran al extensionismo con un enfoque crítico-emancipatorio.

Los aportes al modelo contrahegemónico que promueve la UNICAM-SURI, en cierta medida, trascienden la zona de influencia de la propia Universidad. En ese sentido, esta intenta contribuir a la construcción de soberanía alimentaria a través de la formación de sus propios técnicos y académicos que construyen conocimiento desde sus propios saberes. La idea que subyace es que ellos estarán formados para defender y dotar de herramientas a los campesinos, campesinas, agricultores y trabajadores rurales para que puedan luchar por sus territorios dentro y fuera de Santiago del Estero. Esta Universidad, al igual que el movimiento campesino del cual es parte, propone un modelo alternativo que fomenta la agroecología como sistema productivo y forma de vida. A su vez, la agroecología se está consolidando y construyendo junto con los movimientos y procesos sociales y políticos, por lo tanto podemos plantear que es un proceso de coevolución que busca equilibrar los sistemas naturales y sociales. En ese marco, en la UNICAM-SURI se producen semillas y bioinsumos propios. $Y$ respecto de las semillas, su resguardo y provisión a escuelas, talleres, INTA, otros agricultores, etc., además de fomentar la trascendencia del proyecto más allá de la zona de influencia de la Universidad, aporta al cuidado de la biodiversidad.

Es perentorio asumir el compromiso político de reflexionar sobre el aporte de la agroecología a los Objetivos de Desarrollo Sustentable formulados por la Organización de las Naciones Unidas y ubicar en el primer lugar de las agendas políticas el derecho a una alimentación sostenible y saludable. Se encuentra en manos de los formuladores de políticas públicas reconocer el papel central de las explotaciones agropecuarias familiares de pequeña escala o campesinas, potenciar la producción agroecológica para alcanzar la soberanía alimentaria e incentivar las actividades de los movimientos sociales vinculados con estos procesos pedagógicos que surgen y se nutren desde el extensionismo crítico-emancipatorio.

\section{Referencias bibliográficas}

Altieri, M. (2007). La agroecología como alternativa sostenible frente al modelo de agricultura industrial. Realidad Económica, (229), 75-93. 
(Coord.) (1999). Agroecología: Bases científicas para una agricultura sustentable. Nordan-Comunidad. Argumedo, A. (1996).Los silencios y las voces en América Latina. Notas sobre el pensamiento nacional y popular. Ediciones del Pensamiento Nacional.

Arrillaga, H.; Marioni, L. (2015). La interacción de la universidad con su entorno y los modelos de contribución al desarrollo. Ciencias Económicas, (12)2. 19-41. http://bibliotecavirtual.unl.edu.ar/publicaciones/index.php/ CE/article/view/5461/8174

Baraldo, N. (2009). Movimientos sociales y educación en Argentina: una aproximación a los estudios recientes. Eccos Revista Científica, 11(1), 77-93.

- (2010). Educación en y desde los movimientos sociales: ¿ nuevo objeto y nuevos abordajes en educación? Cuadernos de educación, (8).

Burgos, A. (2013).Movimientos sociales, resistencia y educación La escuela agroecología del Movimento Campesino de Santiago del Estero/vía Campesina. Tesis de Maestría en Educación, Pedagogías críticas y problemáticas socioeducativas. FIUBA.

(2017). Movimientos sociales, resistencia y educación: la escuela agroecológica del Movimiento Campesino de Santiago del Estero/Vía Campesina. Tesis para obtener el grado de Magister de la Universidad de Buenos Aires en Educación, Pedagogías críticas y problemáticas socioeducativas. http://repositorio.filo.uba. ar:8080/bitstream/handle/filodigital/4271/uba_ffyl_t_2013_891632.pdf?sequence=1\&isAllowed=y

De Dios, R. (2006). Expansión agrícola y desarrollo local en Santiago del Estero. Ponencia de la VII Reunión de la Asociación latinoamericana de Sociología Rural. ALASRU. Quito, Ecuador.

- (2012). Ordenamiento territorial e inclusión social en Santiago del Estero. Realidad económica, (268).

Di Ciocco, C.; Tsakoumagkos, P.; Iñíguez, M. y Penón E. (2017). Conductas agropecuarias y problemática ambiental en la pradera bonaerense. Cuatro casos en el noreste provincial. Red Sociales. Revista del Departamento de Ciencias Sociales, (04) 02, 20-36.

Domínguez, D. y Sabatino, P. (2006). Con la soja al cuello: crónica de un país hambriento productor de divisas. CLACSO.

Etxezarreta, M. (2006). La agricultura española en la era de la globalización. En Tendencias de evolución de la agricultura al principio del siglo XXI. Ministerio de Agricultura Pesca y Alimentación (MAPA). Madrid.

FAO (Organización de las Naciones Unidas para la Alimentación y la Agricultura) (2017). The future of food and agricultura. Trends and challenges. http://www.fao.org/3/a-i6583e.pdf

FIAN (Food First Information y Action Network) (2003). http://www.nuevasion.com.ar/archivos/6742

Freire, P. (1973). ¿Extensión o Comunicación? La concientización en el medio Rural. Siglo XXI Editores.

García, M. (2015). Agriculturización y pampeanización argentina. Políticas públicas para el desarrollo rural y territorial en la provincia de Santiago del Estero. Congreso de la Asociación de Economía Agraria Argentina.

García, M. y Puppi, N. (2007). Tenencia de la tierra y tecnología en productores de soja de Santiago del Estero. Comparación con productores bonaerenses. Revista Facultad de Agronomía UBA, 27, 155-171.

García, M.; González, M. C; y Román, M. E. (2016). Una visión exploratoria del desmonte para distintos tipos de productores. En tres departamentos de la provincia de Santiago del Estero (Capítulo 5, pp. 103-124). En Román, M. y González, M. C. (Coords.). Transformaciones agrarias argentinas durante las últimas décadas. Una visión desde Santiago del Estero y Buenos Aires. Coordinadoras. FAUBA.

García, M.; Villa, S. y Calefato, N. (2018). Controversias entre los actores sociales involucrados en la problemática de los agricultores familiares de Santiago del Estero. X Congreso ALASRU. Montevideo.

Gazzano, I.; Achkar, M.; Apezteguía, E.; Ariza, J.; Gómez Perazzoli, A. y Pivel, J. (2021). Ambiente y crisis en Uruguay. La agroecología como construcción contrahegemónica. Revista de Ciencias Sociales, 34(48), 13-40. 
Giarracca, N. (2006). Territorios en disputa: los bienes naturales en el centro de la escena. Realidad Económica, 217, 51-68.

Giarracca, N. y Teubal, M. (2008). Del desarrollo agroindustrial a la expansión del "agronegocio": el caso argentino. En Mançano Fernández, B. (Coord.). Campesinado y Agronegocios en América Latina. CLACSO-ASDI.

Gliessman, S.; Friedmann, H. y Howars, P. (2019). Agroecology and Food Sovereignty. The political economy food.

Gluz, N. (2010).De la autonomía como libertad negativa a la construcción de proyectos colectivos: la escolarización en los movimientos sociales. En Feldfeber, M. (Coord.).Autonomía y gobierno de la educación. Perspectivas, antinomias y tensiones. Aique.

(2011). Movimientos sociales y derecho a la educación. XXVIII Congreso Internacional de ALAS. UFPE. Recife

González González Gil, R. y González Fernández-Larrea, M. (2003). Extensión universitaria: principales tendencias en su evolución y desarrollo. Revista Cubana de Educación Superior.

Guaglianone, A. (2001). Análisis y evolución del impacto del modelo de desarrollo obrajero-forestal en el Chaco santiagueño. El caso de los Jauríes. XXIII International Congress of the Latin American Studies Association. Washington.

Guelman, A. (2012). Formación para el trabajo en una empresa recuperada: Trabajo territorial y formación política. Revista del IICE, (31), 69-82.

Guelman, L. A. (2014). Educación popular y pedagogía descolonizadora en el MOCASE-VC. Intersticios de la política y la cultura. Intervenciones latinoamericanas, 3(6), 35-49.

Guhur, D. y Toná, N. (2017). Diccionario de educación en el campo: Agroecología (pp. 67-78). Batalla de Ideas. Guzmán Casado, G.; González de Molina, M. y Sevilla Guzmán, E. (2000). Introducción a la agroecología como desarrollo rural sostenible. Mundi-Prensa.

Herrera-Jaramillo, M.; Méndez, Y.; Tobón, G. y Sierra, A. (2016). Ni pequeño productor, ni agricultor familiar, soy campesino. En Beltrán, A.; Osorio, F.; Uribe, G.; Ferro, J.; Herrera, J.; Cadavid, M.; Castillo, O. (Eds.). Dime qué Paz quieres y te diré qué campo cosechas: reflexiones sobre lo rural en los diálogos de La Habana (pp. 149-176)

Informe Santiago del Estero (2003). Ministerio de Justicia, Seguridad y Derechos Humanos de la Nación Argentina.

Juárez, P. (2016). Del Granero del mundo a la huerta: Aprendizajes de Política Tecnológica para la Soberanía Alimentaria en Argentina (2001-2012). En Thomas, H. y Santos, G. (Coords.). Tecnología para Incluir. Lenguaje Claro.

Juárez, P. (Coord.); Becerra, L.; Ramella, S.; Ayala, S.; Avellaneda, N.; De León, S.; Bidinost, A.; Ferreyra, I.; Buenaventura, B. y Moreyra, A. (2016). Gestión de saberes y conocimientos científico tecnológicos para la resolución de problemáticas de agua en Argentina. Relevamiento, sistematización y análisis de aprendizajes de gestión, tecnologías y normativas. Programa SEDCERO. Buenos Aires.

Juárez, P.; Trentini, F. y Becerra, L. (2018). Transformative Social Innovation for Food Sovereignty: The Disruptive Alternative. The International Journal of Sociology of Agriculture and Food, 24(3).

Korol, C. (2007). La formación política de los movimientos populares latinoamericanos. Observatorio Social de América Latina, 8(22).

López Geronazzo, L. N. y López, M. L. (2018). Congresos nacionales de extensión universitaria: lógicas de extensión y problematización de los organizadores "comunidad", "sociedad" y "desarrollo social". +E Revista de Extensión Universitaria, 8(9), 12-75. http://hdl.handle.net/11336/91871 
López, M. L. (2020). Teoría y metodología de la extensión universitaria. El caso Argentino. El Siku. Macedo, M. L. (2020). Universidad y Escuela Campesina: Educación Popular y Pedagogía Descolonial en el Mocase Vía Campesina, Argentina. Tesis de maestría Programa de Pós-Graduação Políticas Públicas e Desenvolvimento. Biblioteca Digital de Dissertações e Teses, http://dspace.unila.edu.br/123456789/5901

Mato, D. A. (2015). Pueblos indígenas, Estados y educación superior: Aprendizajes de experiencias en varios países de América Latina potencialmente útiles a los procesos en marcha en Argentina. Cuadernos de Antropología Social, (41), 5-23. Instituto de Ciencias Antropológicas. Facultad de Filosofía y Letras. Universidad de Buenos Aires. http://hdl.handle.net/11336/55266

McMichael, P. (2005). Global development and the corporate food regime. En Buttle, F. y McMichael, P. (Eds.) (2005). New Directions in the Sociology of Global Development (pp. 265-299). Emerald Group Publishing Limited.

Mejía, M. R. (2014). La educación popular: una construcción colectiva desde el sur y desde abajo. Education Policy Analysis Archives/Archivos Analíticos de Políticas Educativas, 22, 1-31.

Michi, N. (2010).Movimientos campesinos y educación El Movimiento de los Trabajadores Rurales Sin Tierra y el Movimiento Campesino de Santiago del Estero VC. El Colectivo.

MNCI (Movimiento Nacional Campesino Indígena) (2010). Síntesis de la concepción e instancias de formación política del MNCl de Argentina.

MOCASE-VC (2017). Recorriendo caminos polvorientos: Cuadernillo sobre trabajo de base en el MoCaSE-VC. Morello, J.; Pengue, W.; Rodríguez, A. (2005) Etapas de uso de los recursos y desmantelamiento de la biota del Chaco. Fronteras, 4(4), 3-19. FAUBA-GEMAPA.

Navarrete, C. (2017). El enfoque agroecológico: una alternativa al modelo agroindustrial. https://repositoriosdigitales.mincyt.gob.ar/vufind/Record/RepHipUNR_281aa7ccfb327e1da08766bad15d0847

Navarrete, D.; Gallopín, G.; Blanco, M.; Díaz-Zorita, M.; Ferraro, D.; Herzer, H.; Laterra, P.; Morello, J.; Murmis, M.; Pengue, W.; Piñeiro, M.; Podesta, G.; Satorre, E.; Torrent, M.; Torres, F.; Viglizzo, E.; Palomino, H. (2003). Las experiencias actuales de autogestión en Argentina. Nueva Sociedad, 184, 115-128.

Palomino, H. (2004). La Argentina hoy. Los movimientos sociales. Herramienta, 27.

Paz, R. G., y Jara, C. E. (2013). Las nuevas dinámicas de los antiguos conflictos de tierra en regiones extrapampeanas. Voces en el Fénix. Facultad de Ciencias Económicas. UBA. Plan Fénix.

Ploeg, J. D. (2010). Nuevos campesinos. Campesinos e imperios alimentarios. Icaria.

Rigal, L. (1997). La escuela crítica democrática: una asignatura pendiente en los umbrales del siglo XXI. En Imbernón, F. La Educación en el siglo XXI. Los retos en el futuro inmediato. Gráo.

Román, M. E. (2014). Agricultura familiar: concepto, polémicas y algunas cifras para la Argentina. Ciencia Hoy, (140).

Salazar Bondy, A. (1973). Dominación y Extensión Universitarias. Universidades. UDUAL, (51), 11-17.

Salizzi, E. (2013). Expansión de la frontera agropecuaria en el norte de Córdoba: reflexiones en torno a la relación entre "nueva agricultura" y ambiente". CD Rom. VIII Jornadas Interdisciplinarias de Estudios Agrarios y Agroindustriales. Facultad de ciencias Económicas. UBA.

Santos, C. F. D.; Siqueira, E. S.; Araújo, I. T. D. y Maia, Z. M. G. (2014). A agroecologia como perspectiva de sustentabilidade na agricultura familiar. Ambiente \& Sociedade, 17(2), 33-52.

Segrelles Serrano, J. A. (2010). Reformas agrarias en América Latina...y algo más. Congreso Reformas Agrarias y Gestión de los Recursos Naturales en África y América Latina. Lleida.

Serna Alcántara, G. A. (2007). Misión social y modelos de extensión universitaria: del entusiasmo al desdén. Revista Iberoamericana de Educación, 43(3), 1-7. https://rieoei.org/RIE/article/view/2324 
Sevilla Guzmán, E. (2011). Sobre los orígenes de la agroecología en el pensamiento marxista y libertario. AGRUCO, Plural editores, CDE, NCCR.

Svampa, M. (2012). Consenso de los commodities, giro ecoterritorial y pensamiento crítico en América Latina. OSAL, 13(32), 15-38.

Teubal, M. (2002). Globalización y Nueva Ruralidad en América Latina. En Giarraca, N. (Comp.). ¿Nueva Ruralidad en América Latina? (pp. 45-65). CLACSO.

Toledo, V. M. (2011). La agroecología en Latinoamérica: tres revoluciones, una misma transformación. Agroecología, 6, 37-46.

Tommasino, H. y Cano, A. (2016). Modelos de extensión universitaria en las universidades latinoamericanas en el siglo XXI: tendencias y controversias. Universidades, 66(67), 7-24. http://www.redalyc.org/articulo. oa? id $=37344015003$

Torres Carrillo, A. (2008). La sistematización de experiencias: aporte de la educación popular para una nueva universidad. Diálogo de saberes, (2). Universidad Bolivariana de Venezuela.

Vidal Torró I, J. P. (2015). Formulación del proyecto de radios comunitarias campesinas del MOCASE-VC en Santiago del Estero, Argentina. http://www.upv.es/entidades/CCD/infoweb/ccd/info/proyecto_pablo.pdf

Wezel, A. and Soldat V. (2009). A quantitative and qualitative historical analysis of the scientific discipline of agroecology. International Journal of Agricultural Sustainability, 7(1), 3-18.

Wezel, A.; Bellon, S.; Doré, T.; Francis, C.; Vallod, D. and David, C. (2009). Agroecology as a science, a movement, and a practice. Agronomy for Sustainable Development, 29(4), 503-515.

Zeolla, N. H. (2012). La teoría clásica de la renta diferencial. Una aproximación al papel de la renta del cultivo de soja en el periodo post-convertibilidad. Estudios de Economía Política y Sistema Mundial (16). https:// www.centrocultural.coop/revista/16/lateoria-clasica-de-la-renta-diferencial-una-aproximacion-al-papel-dela-renta-del.

Zibechi, R. (2003). Los movimientos sociales latinoamericanos: tendencias y desafíos. En OSAL, (9) CLACSO http://bibliotecavirtual.clacso.org.ar/ar/libros/osal/osal9/zibechi.pdf

(2005). La educación en los movimientos sociales. Programa de las Américas. Silver City, NM: International Relations Center. www.americaspolicy.org 